\title{
Chlorpyrifos Induces Delayed Cytotoxicity after Withdrawal in Primary Hippocampal Neurons through Extracellular Signal-Regulated Kinase Inhibition
}

\author{
De-Hong TAN, ${ }^{a, b}$ Shuang-Qing PENG, ${ }^{*, a}$ Ying-Liang Wu,,${ }^{b}$ Yi-Mei WANG, ${ }^{a}$ Chun-Feng Lu,,$b$ \\ Wei Ding, ${ }^{a}$ Qiao-Xu WANG, ${ }^{a}$ and Chang-Hui Y AN $^{a}$ \\ ${ }^{a}$ Evaluation and Research Center for Toxicology, Institute of Disease Control and Prevention, Academy of Military \\ Medical Sciences; 20 Dongdajie Street, Fengtai District, Beijing 100071, P. R. China: and ${ }^{b}$ Department of Pharmacology, \\ College of Life Science and Biopharmaceutics, Shenyang Pharmaceutical University; Shenyang 110016, P. R. China. \\ Received March 13, 2009; accepted July 6, 2009; published online July 24, 2009
}

In this study, the delayed effect and related mechanism after chlorpyrifos (CPF) withdrawal was studied in primary rat hippocampal neurons. The results showed that $10 \mu_{\mathrm{M}} \mathrm{CPF}$ induced no detectable cytotoxicity during $96 \mathrm{~h}$ continuous exposure while its withdrawal after $48 \mathrm{~h}$ exposure induced evident cytotoxicity, as indexed by decreased methyl thiazolyl tetrazolium (MTT) metabolism, increased loss of neurons immunostained by neuronspecific enolase (NSE) antibody, and the increased terminal deoxynucleotidyl transferase-mediated deoxyuridine triphosphate nick end labeling (TUNEL) positive cell rate in the following $24 \mathrm{~h}$ and $48 \mathrm{~h}$ incubation in the absence of CPF. Extracellular signal-related kinase (ERK)1/2 activation by phosphorylation was observed and persisted during CPF exposure. However, CPF withdrawal after $48 \mathrm{~h}$ exposure led to inhibition of ERK1/2 phosphorylation. Carbacol and nerve growth factor (NGF), which are ERK1/2 activators, protected the neurons after CPF withdrawal, while atropine and PD98059, which are ERK1/2 inhibitors, exacerbated the cytotoxicity, indicating the involvement of inhibition of ERK1/2 phosphorylation in CPF-induced delayed cytotoxicity. In conclusion, CPF withdrawal after exposure induced delayed cytotoxicity in cultured neurons. Inhibition of ERK1/2 phosphorylation was found to be related to the delayed cytotoxicity. This finding may provide a new insight into the toxicological mechanism of organophosphorus pesticides, especially chronic organophosphate-induced neuropsychiatric disorder characterized by delayed occurrence.

Key words organophosphorus; pesticide; chlorpyrifos; acetylcholinesterase; hippocampal neuron

Organophosphorus (OP) pesticides are extensively used for control of insects around the home and in agricultural practice. However, they can pose a threat to public health. OP pesticides are known to cause millions of acute poisoning cases per year around the world. ${ }^{1)}$ Besides acute poisoning, OP pesticide exposure causes chronic neurological consequences, especially those related to poor neuropsychiatric changes or neurobehavioral performances in some cognitive domains or mood domain. ${ }^{2}$ The chronic neurological consequence is called chronic OP-induced neuropsychiatric disorder (COPIND) or organophosphorus ester-induced chronic neurotoxicity (OPICN) ${ }^{3-5)}$ which can occur not only after acute OP pesticide intoxication but after long-term low-level exposure.

The mechanism of acute toxicity of OP pesticide has been fully studied and understood. The symptom after absorption of OP pesticide is acute cholinergic syndrome due to inhibition of acetylcholinersterase (AChE) that degrades acetylcholine (ACh) ${ }^{6}$ ) By contrast, the mechanism of chronic neurological consequence or COPIND is not very clear now.

COPIND is usually long-lasting. Agricultural workers performed worse on tests of cognitive and psychomotor function in 2 years after OP pesticide intoxication., ${ }^{2,7)}$ In experimental studies, cognitive deficit was found to last days to months after cessation of OP pesticides exposure. ${ }^{8,9}$ The long-lasting neuropsychiatric changes suggest that the central nervous system has been permanently damaged.

COPIND is sometimes delayed in occurrence, independent of acute toxicity. ${ }^{10)}$ It was found among sheep dippers that long term ( 2 months later) neurotoxic effects occurred inde- pendent of symptoms arising from acute OP pesticides exposure. Only a slight increase in immediate effects but a significant increase in long-term effects were detected compared to the controls ${ }^{11)}$; Women greenhouse workers were found to show no abnormal neurological changes during the working season despite their contact with OP pesticides. However, abnormal neurological changes occurred after the working season. ${ }^{12)}$ Song $e t ~ a l .{ }^{13)}$ found that neurotoxicity appeared after several days' delay upon termination of OP pesticide exposure to rats; Prendergast et al. ${ }^{14)}$ found the impairment of working memory after OP pesticide withdrawal. These findings suggest that COPIND may not derive from the direct effect of OP pesticide to some extent. The mechanism of delayed neurotoxicity remains little known.

In vitro studies may help elucidate the mechanism of OP pesticide neurotoxicity. In this study, the delayed cytotoxicity of chlorpyrifos (CPF) was probed in cultured rat hippocampal neurons. CPF was chosen since it has been a widely used OP pesticide and confirmed to induce long-term neurotoxicity in vivo ${ }^{8,15,16)}$ and neurotoxicity in vitro. ${ }^{17,18)}$ Primary hippocampal neurons were used, for hippocampus was the cognition-related brain region and vulnerable to CPF insult. ${ }^{16,19,20)}$

In the present study, no detectable cytotoxicity was found in the cultured hippocampal neurons during continuous exposure to $10 \mu \mathrm{M} C P F$ for $96 \mathrm{~h}$, but CPF withdrawal after $48 \mathrm{~h}$ exposure induced evident cytotoxicity. Inhibition of extracellular signal-regulated kinase (ERK)1/2 phosphorylation was found to be involved in the mechanism of delayed cytotoxicity. 


\section{MATERIALS AND METHODS}

Chemicals CPF (99.3\%) was purchased from the Institute for the Control of Agrochemicals, Ministry of Agriculture, PR China. Fetal bovine serum (FBS) was obtained from HYCLONE (Utah, U.S.A.). Dulbecco's modified Eagle's medium (DMEM)/F12 medium, B-27, and HEPES were obtained from GIBCO/Invitrogen Corp. (Carlsbad, CA, U.S.A.). Trypsin, poly-L-lysine, cytosine arabinoside, methyl thiazolyl tetrazolium (MTT), carbacol, atropine, NGF (nerve growth factor- $\beta$ from rat) were obtained from Sigma-Aldrich Co. (St. Louis, MO, U.S.A.). PD98059 was from Calbiochem (Arlington Heights, IL, U.S.A.). Antibodies to neuron specific enolase (NSE), ERK2 and phosphorylated (p)-ERK1/2 were purchased from Santa Cruz Biotechnology Inc. All other chemical reagents were of analytical grade unless indicated otherwise.

Primary Hippocampal Neuron Culture All experiments using live animals were carried out in accordance with the Declaration of Helsinki (World Medical Association 2002) and with the provisions of Laboratory Animal Administration Ordinance issued by the State Science and Technology Committee of the People's Republic of China (P. R. China). Hippocampal neurons were prepared as described by Zhang et al. ${ }^{21)}$ with slight modification. Briefly, hippocampi from newborn (0d) Sprague-Dawley rats (Laboratory Animal Center, Academy of Military Science, P. R. China) were rapidly dissected into small pieces and incubated in $\mathrm{Ca}^{2+}$. and $\mathrm{Mg}^{2+}$-free Hanks' balanced salt solution with $2.5 \mathrm{mg} / \mathrm{ml}$ trypsin for $20 \mathrm{~min}$ at $37^{\circ} \mathrm{C}$. The reactions were terminated by adding DMEM/F12 medium containing 10\% heat-inactivated FBS and triturated with a fire-polished Pasteur pipette. After centrifugation for $5 \mathrm{~min}$ at $800 \times \boldsymbol{g}$, the cells were resuspended in DMEM/F12 medium supplemented with $10 \%$ FBS, 2\% B-27, $0.5 \mathrm{~mm}$ L-glutamine, $100 \mathrm{U} / \mathrm{ml}$ penicillin, and $100 \mathrm{mg} / 1$ streptomycin. For MTT assay, $2.0 \times 10^{4}$ cells were plated on each well of poly-L-lysine-coated 96-well plates. For Western blot assay, $2.0 \times 10^{6}$ cells were plated on each well of poly-L-lysine-coated 6-well plates. For immunocytochemical staining and TUNEL [terminal deoxynucleotidyl transferase mediated deoxyuridine triphosphate (dUTP) nick end-labeling] assay, a 13-mm glass coverslip coated with poly-L-lysine was placed in the middle of each well in 12well plates, and $1 \times 10^{5}$ cells were plated onto each well. The medium was entirely replaced by the same volume of the previous DMEM/F12 medium without FBS after $24 \mathrm{~h}$ incubation. Cytosine arabinoside $(10 \mu \mathrm{M})$ was added to the medium on the third and fourth day to inhibit non-neuronal cell division. The culture medium was replaced every 2 or 3 d. Neuronal composition was identified and estimated by immunohistochemical staining with the antibody against NSE on the 7th day (DIV 7).

Drug Treatment Hippocampal neurons were cultured for $7 \mathrm{~d}$ before CPF treatment. CPF was dissolved in ethanol (final concentration: $0.05 \%$, this level of ethanol without cytotoxicity) and freshly prepared for each experiment.

For continuous exposure, the cultures were treated with various concentrations of $\mathrm{CPF}$ for indicated time. For withdrawal study, the cultures were treated with $10 \mu \mathrm{M} C P F$ for $48 \mathrm{~h}$ before CPF was removed by replacing all the culture medium. Fresh medium containing vehicle ethanol instead of
CPF was used for the following incubation. To study the effect of ERK1/2 activators and inhibitors, atropine, PD98059, NGF or carbacol was added after CPF withdrawal to the fresh medium for the following incubation.

MTT Metabolism Assay This was done as described but with some modification. ${ }^{21)}$ Briefly, $20 \mu \mathrm{l}$ of MTT $(5 \mathrm{mg} / \mathrm{ml})$ was added into each well of the culture in the 96well culture plate. After $4 \mathrm{~h}$ incubation, the culture medium was discarded, $150 \mu$ l dimethyl sulfoxide was added and the culture plate was shaken. The optical density (OD) of each well was measured at $570 \mathrm{~nm}$ with an ELISA Reader (Mutiskan MK3, Thermo Electronic). The results are expressed as the ratio of the treated to control OD values at indicated time.

NSE Immunohistochemical Staining Assay After being fixed with $4 \%$ paraformaldehyde, the cultures were preincubated in $5 \%$ bovine serum in phosphate bufferedsaline (PBS) supplemented with $0.2 \%$ Triton-X 100 for $1 \mathrm{~h}$ at room temperature, followed by incubation in primary antibody (anti-NSE, 1:500 dilution, in PBS containing 0.2\% Triton-X 100) overnight at $4{ }^{\circ} \mathrm{C}$. On the next day, binding of primary antibody was detected with a biotinylated secondary antibody using the $\mathrm{ABC}$ kit according to the instructions of the manufacturer. 3,3-Diaminobenzidine tetrahydrochloride (DAB) was used as the substrate. The NSE immunostained cultures were then visualized and analyzed microscopically.

TUNEL Assay DNA damage in dying cells was identified using In Situ Cell Death Detection Kit, POD (Roche Diagnostics Gmbh, Penzberg, Germany) according to the manufacturer's instruction manual, with DAB as the reaction substrate to generate a brown reaction product. Negative controls were performed by substituting distilled water for TdT in the working solution. TUNEL assay was then analyzed microscopically. Using a $10 \times$ reticule and $40 \times$ objective, the numbers of TUNEL positive cells and total cell numbers were counted in five randomly chosen fields on each treated or control coverslip. The results are expressed as the ratio of TUNEL-positive cells to total numbers of cells.

Western Blot Assay Western blot was done as described by Zhang et $a l^{21)}$ with some modification. The cells were washed twice with PBS, and then dissolved in $0.1 \mathrm{ml}$ lysis buffer. The cell lysates were centrifuged at $12000 \times \boldsymbol{g}$ for $5 \mathrm{~min}$ at $4{ }^{\circ} \mathrm{C}$, and supernatant fractions were collected on ice. The protein concentrations were determined by BCA protein Assay kits (Sigma Corporation, St. Louis, MO, U.S.A.) and evenly adjusted. The protein extracts were mixed with $5 \times$ sodium dodecyl sulfate (SDS) loading buffer (62.5 mm Tris- $\mathrm{HCl}, \mathrm{pH} 6.8,4 \%$ SDS, $10 \%$ glycerol, $5 \% \beta$ mercaptoethanol and $0.1 \%$ bromophenol blue), and then denatured at $95^{\circ} \mathrm{C}$ for $5 \mathrm{~min} .20 \mu \mathrm{l}$ cell lysate (corresponding to $20 \mu \mathrm{g}$ total protein) was loaded into each lane with $10 \%$ SDS polyacrylamide gel electrophoresis, and then transferred to nitrocellulose membranes. After the run, the blots were blocked with $5 \%$ dried milk for $1 \mathrm{~h}$ at room temperature before being reacted overnight in a cold room with primary antibody. After three short washes, the blots were subsequently incubated for $1 \mathrm{~h}$ at room temperature with HRP-conjugated anti-rabbit immunoglobulin (IgG) or anti-mouse IgG. The blots were then exposed to ECL substrate and film. The primary antibodies for total-ERK2 and phosphorylated ERK1/2 (p-ERK1 and p-ERK2, or phospho-p44/42) were used. Usu- 
ally the blots were first treated with anti-p-ERK1/2 antibody, stripped with stripping buffer (100 mM $\beta$-mercaptoethanol, $2 \%$ SDS, and $62.5 \mathrm{~mm}$ Tris- $\mathrm{HCl}, \mathrm{pH} 6.7)$, and reprobed with anti-ERK2 antibody. Quantification was performed using an image analyzer software (Quantity One ${ }^{\mathbb{R}} 1$-D Analysis Software Version 4.4, BIO-RAD).

Statistical Analysis Data were represented as means \pm standard errors (S.E.s). Statistical significance was assessed by ANOVA with subsequent Dunnett test, and a level of $p<0.05$ was considered statistically significant. All experiments were performed in triplicate.

\section{RESULTS}

Ten Micromolars CPF Continuous Exposure for $96 \mathrm{~h}$ Induces no Detectable Cytotoxicity We first tested the dose- and time-response of direct toxicity of CPF in cultured hippocampal neurons in order to study the withdrawal effect of CPF, and to hunt for a threshold concentration that might induce withdrawal effect without direct toxicity. Before treatment, hippocampal neurons prepared from newborn rats $(\mathrm{P} 0)$ were cultured for $7 \mathrm{~d}$ and identified by NSE immunohistochemical staining (purity of neurons $>90 \%$, data not shown). The neurons were exposed to various concentrations of $\mathrm{CPF}$ or vehicle ethanol ( $0.05 \%$ final concentration) for $24,48,72$ and $96 \mathrm{~h}$, respectively. The cytotoxicity was determined by MTT assay, immunohistochemical staining assay and TUNEL assay.

MTT Assay The MTT assay is based on the colorimetric conversion of the yellow, water-soluble tetrazolium to the blue, water insoluble formazan. This conversion is catalyzed by cellular mitochondrial dehydrogenases. Because the rate of this reaction is proportional to the number of surviving cells and functional mitochondria, MTT assay is widely used to quantify viable cells and to assess mitochondrial function. ${ }^{22)}$ In this study, cultures for MTT assay were treated with cytosine arabinoside to inhibit non-neuronal cell division as in NSE immunohistochemical staining assay. No wonder that cells in MTT assay were mostly neurons. As shown in Fig 1, CPF reduced MTT metabolism in a doseand time-dependent manner within a concentration range of
$30-50 \mu \mathrm{M}$ during up to $96 \mathrm{~h}$ exposure, while $10 \mu \mathrm{M} \mathrm{CPF}$ showed no effect on MTT metabolism ( $p<0.05$ versus control), indicating that CPF at this level has no impact on neuronal viability or mitochondrial function.

Immunohistochemical Staining Assay NSE is a protein expressed heavily in neurons, so neurons can be detected by immunohistochemical staining with anti-NSE antibody. ${ }^{23)}$ As shown in Fig. 2, neurons were NSE immunohistochemical staining positive and showed brown color. Normal neurons exhibited homogeneous and compact morphology with clear neurites while damaged neurons exhibited disappearance of neurites, disrupted membranes, distorted somata, and condensed nuclei. In control cultures tested at the time of CPF exposure for $96 \mathrm{~h}$, most of the cells were normal neurons, with few damaged neurons. CPF exposure increased neuronal loss and damaged neurons in a dose- and time-dependent manner. $10 \mu \mathrm{M} \mathrm{CPF}$ did not evidently increase neuronal loss and damaged neurons.

TUNEL Assay The TUNEL method identifies DNA damaged cells in situ by using terminal deoxynucleotidyl transferase (TdT) transfering labeled-dUTP to these strand breaks of cleaved DNA. ${ }^{24)}$ As shown by representative photomicrographs in Fig. 3 and statistic data in Fig. 4, control cultures consisted of mostly normal neurons with rare

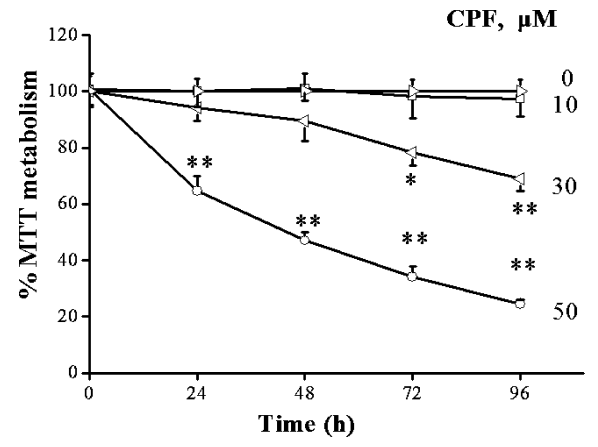

Fig. 1. CPF Decreased MTT Metabolism in Hippocampal Neurons

Hippocampal neurons were prepared from newborn (P0) rats. Seven days after culture in vitro (DIV 7), neurons were treated with various concentrations of CPF (10$50 \mu \mathrm{M})$ for indicated time and MTT metabolism was measured. Data are means and S.E.s of the percentage from corresponding control value. All the data are from three independent experiments, $* p<0.05, * * p<0.01$ versus control at indicated time.
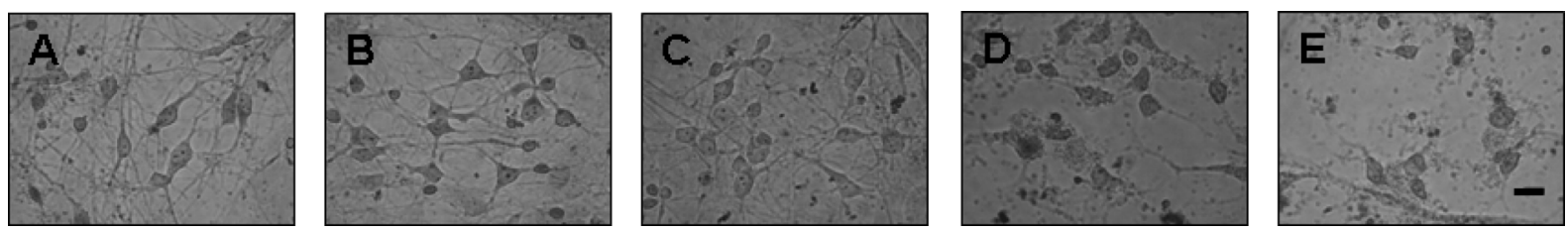

Fig. 2. Representative Photomicrographs in NSE Immunohistochemical Staining Assay for Hippocampal Neurons Exposed to CPF for $96 \mathrm{~h}$

Hippocampal neurons were prepared from newborn (P0) rats. After $7 \mathrm{~d}$ of incubation, the cultured neurons were treated with different concentrations of $\mathrm{CPF}$ for $96 \mathrm{~h}$ and immunohistochemically stained with antibody to NSE. A: control, B: vehicle control, C: $10 \mu \mathrm{M} \mathrm{CPF,} \mathrm{D:} 30 \mu \mathrm{M}$ CPF, E: $50 \mu \mathrm{M}$ CPF. Calibration bar: $100 \mu \mathrm{m}$.
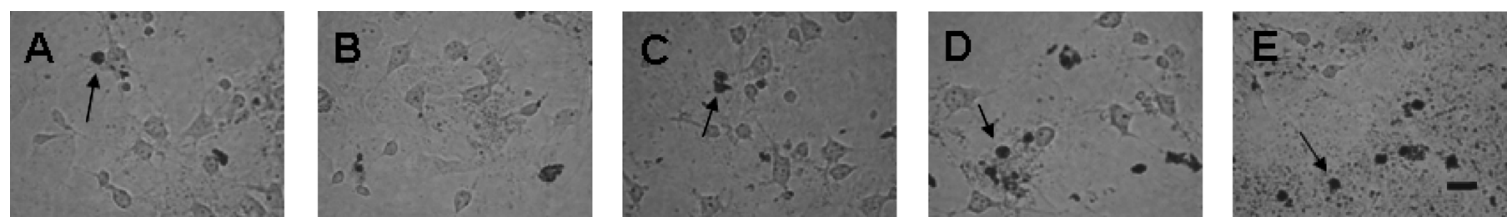

Fig. 3. Representative Photomicrographs in TUNEL Assay for Hippocampal Neurons Exposed to CPF for $96 \mathrm{~h}$

Hippocampal neurons were prepared from newborn (P0) rats. After $7 \mathrm{~d}$ of incubation, the cultured neurons were treated with different concentrations of $\mathrm{CPF}$ for $96 \mathrm{~h}$ and were stained by TUNEL. A: control, B: vehicle control, C: $10 \mu \mathrm{M}$ CPF, D: $30 \mu \mathrm{M} \mathrm{CPF,} \mathrm{E:} 50 \mu \mathrm{M} \mathrm{CPF}$. TUNEL assay shows the presence of damaged DNA in the nuclei as indicated by arrows. Calibration bar: $100 \mu \mathrm{m}$. 


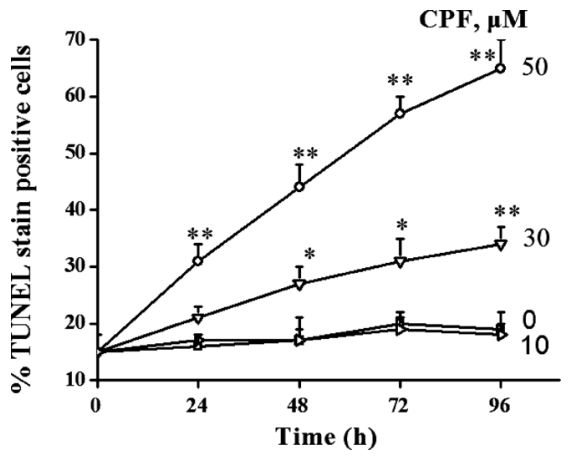

Fig. 4. Quantification of TUNEL Stain Positive Cells Induced by CPF Exposure in Hippocampal Neurons

Hippocampal neurons were prepared from newborn (P0) rats. Seven days after culture in vitro, the neurons were treated with various concentrations of CPF $(0-50 \mu \mathrm{M})$ for indicated time and TUNEL staining was done. Results are averages of three independent experiments. Error bars are S.E.s, $* p<0.05, * * p<0.01$ versus vehicle control (0) at indicated time.

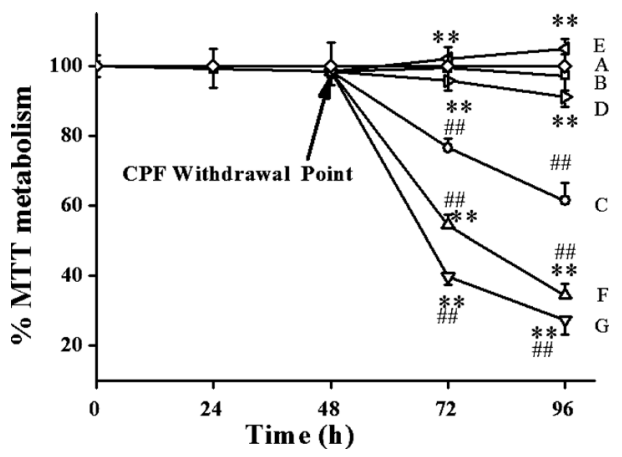

Fig. 5. Effect of $10 \mu \mathrm{M}$ CPF Withdrawal on MTT Metabolism, and Effects of Carbacol, NGF, Atropine or PD98059 on MTT Metabolism after CPF Withdrawal

Time-course of MTT metabolism to vehicle control (A), $10 \mu \mathrm{M}$ CPF continous exposure (B), $10 \mu \mathrm{M}$ CPF exposure for $48 \mathrm{~h}$ followed by CPF withdrawal (C), $10 \mu \mathrm{M}$ CPF exposure followed by CPF withdrawal but exposure to carbacol (D), NGF (E), atropine (F), PD98059 (G). Data are means and S.E.s of the percentage from corresponding control value from three independent experiments conducted in triplicate, $\# p<0.01$ versus vehicle control. $* * p<0.01$ versus $\mathrm{CPF}$ withdrawal.

TUNEL-positive cells. CPF led to increment in TUNEL-positive neurons in a dose- and time-dependent manner within a concentration range of $30-50 \mu \mathrm{M} .10 \mu \mathrm{M}$ CPF did not increase TUNEL positive cells significantly $(p>0.05)$, suggesting that $\mathrm{CPF}$ at this level resulted in no evident DNA damage.

Ten Micromolars CPF Withdrawal after 48 h Exposure Induces Obvious Cytotoxicity It could be seen from the continuous exposure study above that no detectable cytotoxicity was found in cultured hippocampal neurons exposed to $10 \mu \mathrm{M}$ CPF for up to $96 \mathrm{~h}$, so $10 \mu \mathrm{M}$ CPF was used in the following delayed effect study. Hippocampal neurons prepared as before were first exposed to $10 \mu \mathrm{M} \mathrm{CPF}$ for $48 \mathrm{~h}$, then CPF was withdrawn to be replaced by culture medium, and fresh medium without CPF was applied for the following incubation. For continuous CPF exposure control, CPF was added to the fresh medium again for the following incubation. $\mathrm{Cy}$ totoxicity was evaluated as above.

After CPF withdrawal, cytotoxicity became obvious. MTT metabolism was reduced significantly $(76 \%$ and $61 \%$ from control, $24 \mathrm{~h}$ and $48 \mathrm{~h}$ after $\mathrm{CPF}$ withdrawal respectively; $p<0.01$, at any time) (Fig. 5C); immunohistochemical stain-
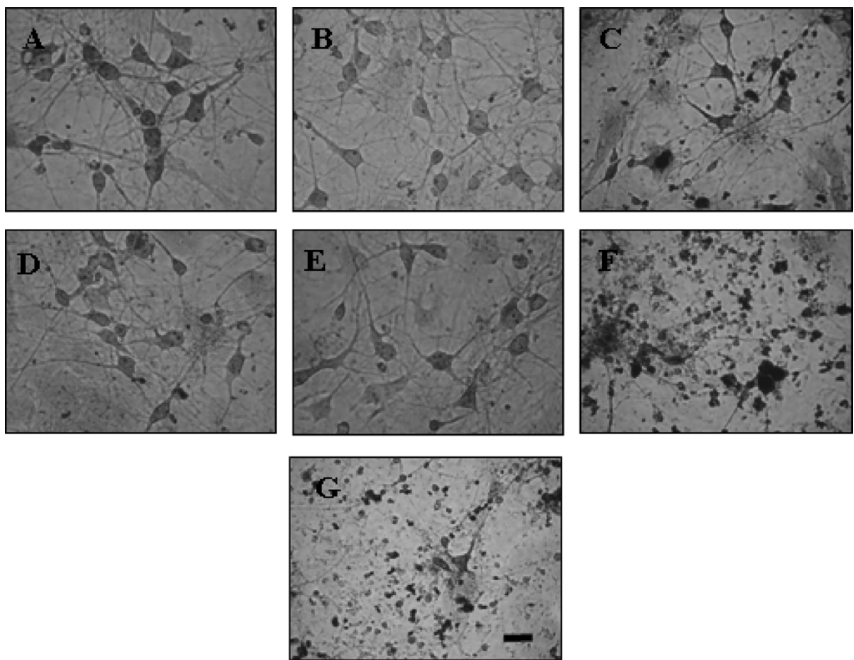

Fig. 6. Representative Photomicrographs in NSE Immunohistochemical Staining Assay after CPF Withdrawal

Hippocampal neurons were prepared from newborn $(\mathrm{P} 0)$ rats after $7 \mathrm{~d}$ incubation, the cultured neurons were treated with vehicle for $96 \mathrm{~h}(\mathrm{~A}) ; 10 \mu \mathrm{M} \mathrm{CPF}$ for $96 \mathrm{~h}(\mathrm{~B}) ; 10 \mu \mathrm{M}$ $\mathrm{CPF}$ for $48 \mathrm{~h}$ followed by CPF withdrawal for $48 \mathrm{~h}(\mathrm{C}) ; 10 \mu \mathrm{M}$ CPF for $48 \mathrm{~h}$ followed by carbacol (D), NGF (E), atropine (F), PD98059 (G) for $48 \mathrm{~h}$. Then the cultures were immunohistochemically stained with antibody to NSE. Calibration bar: $100 \mu \mathrm{m}$.
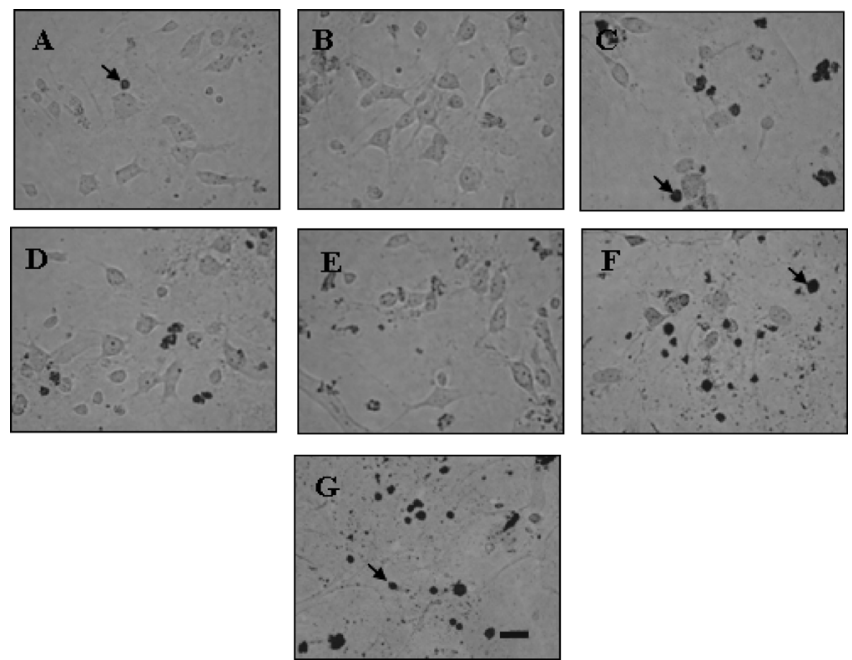

Fig. 7. Representative Photomicrographs in TUNEL Assay after CPF Withdrawal

Hippocampal neurons were prepared from newborn (P0) rats after $7 \mathrm{~d}$ incubation, the

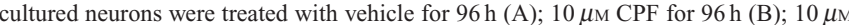
$\mathrm{CPF}$ for $48 \mathrm{~h}$ followed by CPF withdrawal for $48 \mathrm{~h}(\mathrm{C}) ; 10 \mu \mathrm{M}$ CPF for $48 \mathrm{~h}$ followed by carbacol (D), NGF (E), atropine (F), PD98059 (G) for $48 \mathrm{~h}$. TUNEL assay shows the presence of damaged DNA in the nuclei of cultured hippocampal neurons as indicated by arrows. Calibration bar: $100 \mu \mathrm{m}$.

ing assay showed that CPF withdrawal evidently increased neuronal loss and damaged neurons (Fig. 6C); TUNEL staining positive cell rate also increased significantly (17\% to $37 \%$ and $23 \%$ to $45 \%, 24 \mathrm{~h}$ and $48 \mathrm{~h}$ after CPF withdrawal respectively; $p<0.01$ ) (Fig. 7C; Fig. 8C).

Inhibition of ERK1/2 Phosphorylation Involved in CPF Induced Delayed Cytotoxicity As an important member of mitogen activated protein (MAP) kinases superfamily, serving to coordinate the cellular responses to a variety of extracellular stimuli including cholinergic transmitters, ERK $1 / 2$ is activated through a specific phosphorylation cas- 
cade. $^{25,26)}$ Activation of ERK1/2 by phosphorylation has been found involved in the cytotoxicity during CPF exposure. ${ }^{27)}$ To elucidate the underlying mechanism of CPF withdrawalinduced cytotoxicity, p-ERK1/2 was detected before and after CPF withdrawal, and effects of ERK1/2 activators and inhibitors on ERK1/2 phosphorylation and CPF withdrawalinduced cytotoxicity were also examined.

To test the response of $\mathrm{p}$-ERK $1 / 2$ during $10 \mu \mathrm{M}$ CPF continuous exposure and after withdrawal, extracts prepared from hippocampal neurons exposed to $10 \mu \mathrm{M}$ CPF and that after CPF withdrawal for various lengths of time were ana-

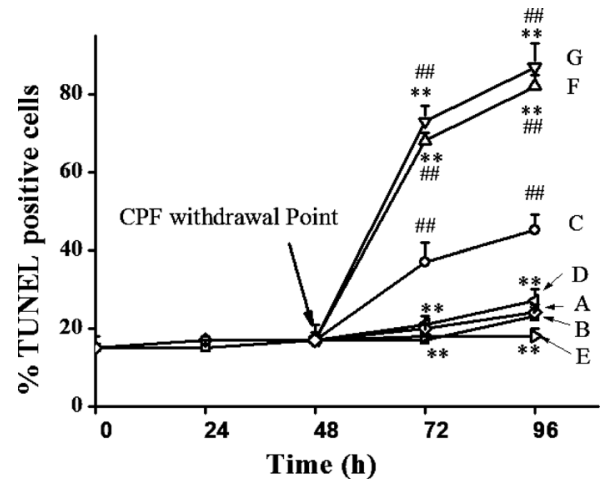

Fig. 8. Quantification of TUNEL Staining Positive Cell Rate Induced by CPF Withdrawal, and CPF Withdrawal But Treatment with Carbacol, NGF, Atropine or PD98059

Hippocampal neurons were prepared from newborn $(\mathrm{P} 0)$ rats. After $7 \mathrm{~d}$ of culture in vitro, cells were exposed to vehicle control (A), $10 \mu \mathrm{M}$ CPF continous exposure (B), $10 \mu \mathrm{M}$ CPF exposure for $48 \mathrm{~h}$ followed by CPF withdrawal (C), $10 \mu \mathrm{M}$ CPF exposure followed by CPF withdrawal but exposure to carbacol (D), NGF (E), atropine (F), PD98059 (G) for the indicated time. Results are averages of three independent experiments. Error bars are S.E.s. \# $p<0.01$ versus vehicle control, $* * p<0.01$ versus $\mathrm{CPF}$ withdrawal. lyzed for the presence of $\mathrm{p}-\mathrm{ERK} 1 / 2$ by Western blot analysis with specialized antibodies.

As shown in Figs. 9A and B, $10 \mu \mathrm{M}$ CPF exposure caused activation of ERK1/2, indicated by the increase of $\mathrm{p}$-ERK1/2. The activation of ERK1/2 began quickly, reached peak at about $0.5 \mathrm{~h}$ after CPF was added to the culture and then decreased gradually to the normal level $24 \mathrm{~h}$ later despite the ongoing CPF exposure.

CPF withdrawal after $48 \mathrm{~h}$ exposure inhibited ERK1/2 activiation, indexed by significant decrement of $\mathrm{p}$-ERK1/2 ( $p<0.01$ for $\mathrm{p}$-ERK2 vs. control, at any time). The decrement showed a time-course manner during the tested time, corresponding to CPF induced delayed cytotoxicity detected above.

ERK1/2 Activators Protected Neurons while ERK1/2 Inhibitors Exacerbated CPF Withdrawal-Induced Cytotoxicity To elucidate the role of ERK1/2 phosphorylation in CPF withdrawal-induced cytotoxicity, the effects of ERK1/2 activators and ERK1/2 inhibitors on p-ERK1/2 and cytotoxicity were tested. The ERK $1 / 2$ activators used here were carbacol and NGF while ERK1/2 inhibitors were atropine and PD98059. After CPF was withdrawn from the culture, $10 \mu \mathrm{M}$ carbacol, $50 \mathrm{ng} / \mathrm{ml} \mathrm{NGF,} 10 \mu \mathrm{M}$ atropine or $20 \mu \mathrm{M}$ PD98059 (all were proved to be without cytotoxicity in cultured hippocampal neurons, data not shown) was added to the culture for the following incubation. p-ERK1/2 was measured $2 \mathrm{~h}$ later, and cytotoxicity was assessed $24 \mathrm{~h}$ and $48 \mathrm{~h}$ after $\mathrm{CPF}$ was removed.

As shown in Figs. 9C and D, both $10 \mu \mathrm{M}$ carbacol and $50 \mathrm{ng} / \mathrm{ml} \mathrm{NGF}$ counteracted the reduction of $\mathrm{p}$-ERK1/2 after CPF withdrawal. p-ERK1/2 after carbacol or NGF treatment was significantly more than that of CPF withdrawal $(p<0.01)$, but was of no significant difference compared with

A

$\mathrm{B}$
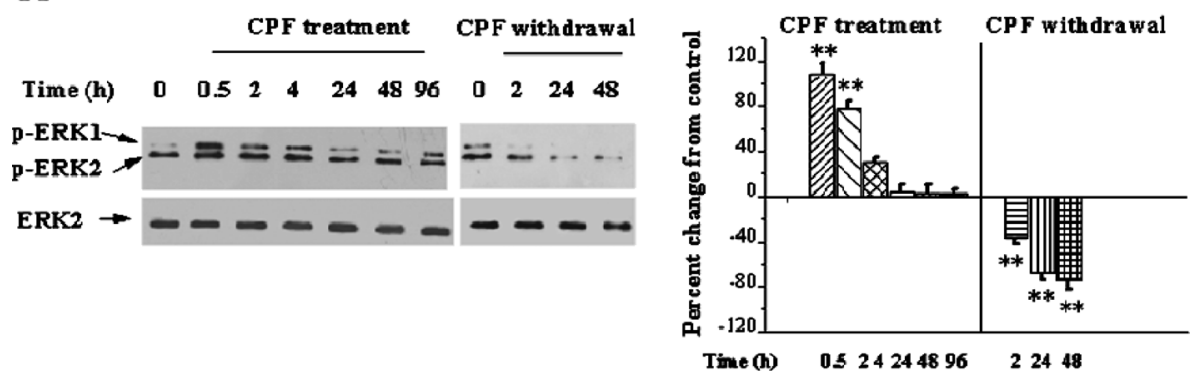

C

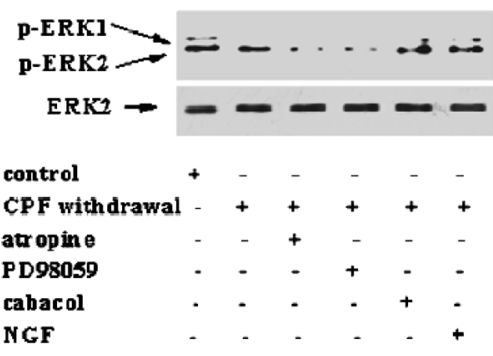

D

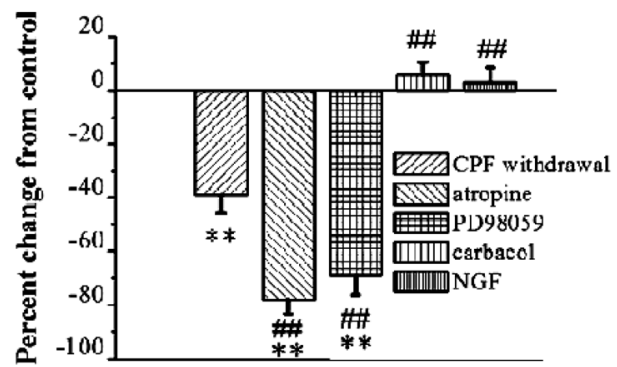

Fig. 9. Role of ERK1/2 Phosphorylation on CPF Withdrawal-Induced Cytotoxicity in Hippocampal Neurons

Cultured hippocampal neurons at DIV 7 were continually treated with $10 \mu \mathrm{M}$ CPF for indicated time, or for $48 \mathrm{~h}$ followed by CPF withdrawal. ERK1/2 phosphorylation was determined by Western blot assay with anti-phosphorylated (p)-ERK1/2 (p-ERK1 and p-ERK2) antibodies, and the level of ERK2 was detected with anti-ERK2 antibody after a stripping procedure for normalization. The resultant Western blot assay pictures were shown in (A). Individual band corresponding to p-ERK2 was quantified by densitometric analysis, and the quantified result expressed as percentage changes and errors to corresponding control $(0 \mathrm{~h})$ was shown in (B). Results are averages of three independent experiments. Error bars are S.E.s, $* * p<0.01$ versus corresponding control. Also the effects of ERK1/2 activators (carbacol and NGF) and inhibitors (atropine and PD98059) on ERK1/2 phosphorylation after CPF withdrawal were examined, the results shown in $(\mathrm{C})$ and (D). $* * p<0.01$ versus corresponding control. \# $p<0.01$ versus $\mathrm{CPF}$ withdrawal. 
that of control $(0 \mathrm{~h})(p>0.05)$. While both $10 \mu \mathrm{M}$ carbacol and $50 \mathrm{ng} / \mathrm{ml}$ NGF protected neurons from damage by CPF withdrawal, there was no obvious cytotoxicity found in each MTT assay ( $p>0.05$ versus control, at any time, Figs. $5 \mathrm{D}, \mathrm{E}$ ), immunohistochemical staining assay (Figs. 6D, E) and TUNEL assay ( $p>0.05$ versus control, Figs. 8D, E; Figs. 7D, E).

Both $10 \mu \mathrm{M}$ atropine and $20 \mu \mathrm{M}$ PD98059 further reduced p-ERK1/2, which was hardly detectable. Besides, both $10 \mu \mathrm{M}$ atropine and $20 \mu \mathrm{M}$ PD98059 exacerbated CPF withdrawalinduced cytotoxicity, as indexed by sharply-decreased MTT metabolism $(p<0.01$ versus CPF withdrawal, both for atropine and PD98059, at any time. Figs. 5F, G), greatly increased neural loss with only few normal neurons left (Figs. $6 \mathrm{~F}, \mathrm{G})$; and massively TUNEL positive cells existed $(p<0.01$ versus $\mathrm{CPF}$ withdrawal, both for atropine and PD98059, at any time) (Figs. 7F, G; Figs. 8F, G).

\section{DISCUSSION}

The objective of this study was to find out whether CPF could induce delayed cytotoxicity in primary cultured rat hippocampal neurons and to identify the underlying mechanisms. In the present study, while $10 \mu \mathrm{M}$ CPF continuous exposure for $96 \mathrm{~h}$ induced no detectable cytotoxicity, $10 \mu \mathrm{M}$ CPF withdrawal after $48 \mathrm{~h}$ exposure induced obvious cytotoxicity, as evidenced by reduced MTT metabolism, increased neuronal loss, and the increased TUNEL positive cell rate. ERK1/2 activation was found to last long and return to normal despite CPF exposure. ERK1/2 inhibition was found when CPF was withdrawn after $48 \mathrm{~h}$ CPF exposure. Carbacol and NGF, which are ERK1/2 activators, protected the neurons after CPF withdrawal, while atropine and PD98059 as ERK1/2 inhibitors exacerbated the cytotoxicity, indicating that inhibition of ERK1/2 activation plays a key role in CPF induced delayed cytotoxicity.

ERK1/2 is a member of the mitogen activated protein kinase (MAPK) family, and an important mediator of signal transduction processes that serve to coordinate the cellular responses to a variety of extracellular stimuli. ${ }^{25)}$ ERK1/2 is activated through a specific phosphorylation cascade, and participates in a wide range of cellular programs including proliferation, differentiation, apoptosis, and synaptic plasticity $^{27,28)}$ Inhibition of ERK1/2 activation was found to induce neurotoxicity, and activation of ERK1/2 protected neurons. ${ }^{29,30)} \mathrm{NGF}$ which activated ERK1/2 via receptor tyrosine kinase (RTK) during exposure induced inhibition of pERK1/2 and apoptosis after NGF withdrawal in cultured neurons. This inhibition of $\mathrm{p}-\mathrm{ERK} 1 / 2$ was regarded as the mechanism of the apoptosis. ${ }^{31,32)}$ There were also reports that activation of ERK1/2 induced neurotoxicity and inhibition of $\mathrm{p}$ ERK1/2 protected cells. ${ }^{33-35)}$ Although proper activation of ERK $1 / 2$ is needed for neuronal survival, both inadequate and excessive activation can induce cytotoxicity.

In the present study, $10 \mu \mathrm{M}$ CPF exposure caused activation of ERK1/2, but not strong enough to cause cytotoxicity. The activation persisted for hours and returned to normal even in the presence of CPF. CPF withdrawal after $48 \mathrm{~h}$ exposure induced marked inhibition of p-ERK1/2 and delayed cytotoxicity. How activation of ERK1/2 comes to cause inhibition is not clear. Since activation of ERK by stimuli needs energy, ${ }^{36)}$ we speculate that activation of ERK by CPF pretreatment may consume much energy. Upon the termination of long stimulation, there may be no enough energy for activation of ERK, so activation of ERK1/2 turns into inhibition. The role of p-ERK1/2 inhibition in CPF induced delayed cytotoxicity was determined by evaluating the effect of ERK1/2 activators and inhibitors. The ERK1/2 activators in the present study were carbacol and NGF. Carbacol, as a cholinergic agonist, can induce activation of ERK $1 / 2,{ }^{25,36}$ while NGF is a member of a family of neurotrophic factors, known to activate ERK1/2 via RTK. ${ }^{37)}$ Atropine and PD98059 were used as ERK1/2 inhibitors in the present study. Atropine is a cholinergic antagonist and widely used antidote for OP pesticide intoxication, and can inhibit p-ERK1/2 in neurons. ${ }^{25,38)}$ PD98059, an inhibitor of ERK1/2 kinase (MEK), is often used as an inhibitor of ERK1/2 pathway. ${ }^{39)}$ In the present study, both NGF and carbacol augmented activation of ERK1/2 that was otherwise inhibited after CPF withdrawal, and protected the neurons, while both atropine and PD98059 further inhibited p-ERK1/2 and exacerbated CPF withdrawal-induced cytotoxicity. These results confirmed that inhibition of p-ERK1/2 was related to CPF induced delayed cytotoxicity.

Traditionally, much of chlorpyrifos neurotoxicity has been attributed to AChE inhibition via its active metabolite chlorpyrifos-oxon. However, recent studies suggest that additional mechanisms may also be at work. Chlorpyrifos is about two to three orders of magnitude less potent than chlorpyrifosoxon in inhibition of brain AChE activity, but chlorpyrifos was only slightly less potent than chlorpyrifos-oxon in inducing cortical neuron apoptosis. ${ }^{18)}$ Inhibition of macromolecule and protein synthesis was believed to be related to chlorpyrifos neurotoxicity. ${ }^{13)}$

The finding that CPF withdrawal after a period of exposure induced inhibition of p-ERK1/2 and delayed cytotoxicity in vitro not only provides new clues to explaining the mechanism of CPF cytotoxicity, but reminds us that cessation of OP exposure after a period of low level exposure may impair the nervous system and disturb the ERK system, leading to delayed neurotoxicity, especially cognitive disorder. More in vivo experiments are needed.

\section{CONCLUSION}

In the present study, $\mathrm{CPF}$ at concentration without direct cytotoxicity during continuous exposure can induce cytotoxicity after CPF withdrawal in cultured neurons, and inhibition of ERK1/2 phosphorylation is found to be related to the delayed cytotoxicity. The result sheds light on the mechanism of COPIND which is sometimes delayed in occurrence.

Conflict of Interest Statement I declare no conflict of interest.

Acknowledgments This work was supported by the China National Scientific Supporting Program (2006 BAK02A02) and the Grant of National Natural Science Foundation of China (30800929). The contents of this manuscript are solely the responsibility of the authors and do not necessarily represent the official views of the sponsor. The authors thank senior technician Ai-Shi Ding for technical assistance. 


\section{REFERENCES}

1) Raheja G., Gill K. D., Toxicol. Ind. Health, 23, 25-37 (2007).

2) Wesseling C., Keifer M., Ahlborn A., McConeel R., Moon J., Risenstock L., Hogstedt C., Int. J. Occup. Health, 8, 27-34 (2002).

3) Jamal G. A., Adverse Drug React. Toxicol. Rev., 16, 133-170 (1997).

4) Abou-Donia M. B., Arch. Environ. Health, 58, 484- 497 (2003).

5) Colosio C., Tiramani M., Maroni M., Neurotoxicology, 24, 577-591 (2003).

6) Storm J. E., Rozman K. K., Doull J., Toxicology, 150, 1-29 (2000).

7) Rosenstock L., Keifer M., Daniell W., McConnell R., Claypoole K., Lancet, 338, 223-227 (1991).

8) Cañadas F., Cardona D., Dávila E., Sánchez-Santed F., Toxicol. Sci., 85, 944-951 (2005).

9) Kassa J., Koupilova M., Vachek J., Pharmacol. Biochem. Behav., 70, $175-179$ (2001)

10) Kamel F., Hoppin J. A., Environ. Health Perspect., 112, 950-958 (2004).

11) Stephens R., Spurgeon A., Berry H., Neurotoxicol. Teratol., 18, 449453 (1996).

12) Bazylewicz-Walczak B., Majczakowa W., Szymczak M., Neurotoxicology, 20, 819-826 (1999).

13) Song X., Seidler F. J., Saleh J. L., Zhang J., Padilla S., Slotkin T. A., Toxicol. Appl. Pharmacol., 145, 158-174 (1997).

14) Prendergast M. A., Terry A. V., Buccafusco J. J., Psychopharmacology (Berlin), 129, 183-191 (1997).

15) Sánchez-Santed F., Cañadas F., Flores P., López-Grancha M., Cardona D., Neurotoxicol. Teratol., 26, 305-317 (2004).

16) Terry A. V., Stone J. D., Buccafusco J. J., Sickles D. W., Sood A., Prendergast M. A., J. Pharmacol. Exp. Ther, 305, 375-384 (2003).

17) Zurich M. G., Honegger P., Schilter B., Costa L. G., Monnet-Tschudi F., Toxicol. Appl. Pharmacol., 201, 97-104 (2004).

18) Caughlan A., Newhous K., Namgung U. K., Xia Z., Toxicol. Sci., 78, 125-134 (2004).

19) Eichenbaum H., Neuron, 44, 109-120 (2004).

20) Gron G., Brandenburg I., Wunderlich A. P., Riepe M. W., Neurobiol.
Aging, 27, 78-87 (2006)

21) Zhang M., Wang A., He W., He P., Xu B., Xia T., Chen X., Yang K., Toxicology, 236, 208-216 (2007).

22) Schengrund C., Marangos P., J. Neurosci., 5, 305-311 (2004).

23) Mosmann T., J. Immunol. Methods, 65, 55-63 (1983).

24) Wang W., Iyo A., Miguel-Hidalgoa J., Regunathana S., Zhu M., Brain Res., 1084, 210-216 (2006).

25) Rosenblum K., Futter M., Jones M., Hulme E. C., Bliss T. V., J. Neurosci., 20, 977-985 (2000).

26) Yan G. M., Lin S. Z., Irwin R. P., Paul S. M., Mol. Pharmacol., 47, 248-257 (1995).

27) Atkins C. M., Selcher J. C., Petraitis J. J., Trzaskos J. M., Sweatt J. D., Nat. Neurosci., 1, 602-609 (1998).

28) Robinson M. J., Cobb M. H., Curr. Opin. Cell Biol., 9, 180-186 (1997).

29) Hetman M., Kanning K., Cavanaugh J. E., Xia Z., J. Biol. Chem., 274, 22569-22580 (1999).

30) Szatmari E., Kalita K. B., Kharebava G., Hetman M., J. Neurosci., 27, 11389-11400 (2007).

31) Araki W., Wurtman R. J., Mol. Brain Res., 56, 169-177 (1998).

32) Xia Z., Dickens M., Raingeaud J., Davis R. J., Greenberg M. E., Science, 270, 1326-1331 (1995).

33) Alessandrini A., Namura S., Moskowitz M. A., Bonventre J. V., Proc. Natl. Acad. Sci. U.S.A., 96, 12866-12869 (1999).

34) Murray B., Alessandrini A., Cole A., Yee A., Furshpan E., Proc. Natl. Acad. Sci. U.S.A., 95, 11975-11980 (1998).

35) Stanciu M., Wang Y., Kentor R., Burke N., Watkins S., Kress G., Reynolds I., Klann E., Angiolieri M. R., Johnson J. W., DeFranco D. B., J. Biol. Chem., 275, 12200-12206 (2000).

36) Takagi N., Miyake-Takagi K., Takagi K., Tamura H., Takeo S., J. Biol. Chem., 277, 6382-6390 (2002).

37) Thoenen H., Science, 270, 593-598 (1995).

38) Bowden C. A., Krenzelok E. P., Drug Saf., 16, $9-47$ (1997).

39) Alessi D. A., Cuenda A., Cohen P., Dudley D. T., Saltiel A. R., J. Biol. Chem., 270, 27489-27494 (1995). 